\title{
Síndrome de Fahr: um diagnóstico raro
}

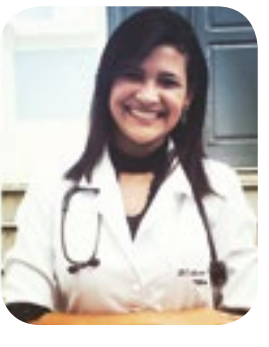

Adriane Souza da Paz', Mateus Santana do Rosário', José César Batista Oliveira Filho', Alina Coutinho Rodrigues Feitosa

Palavras-chave: Síndrome de Fahr, Calcificação dos Núcleos da Base, Hipoparatiroidismo

\section{INTRODUÇÃO}

A doença de Fahr é uma entidade rara e neurodegenerativa, caracterizada pela calcificação bilateral e simétrica dos núcleos da base de associação familiar e idiopática. Quando relacionada a outras doenças, não de origem familiar, é descrita como Síndrome de Fahr. A deposição de cálcio pode ocorrer em outras regiões, como núcleo denteado, cortex cerebral e cerebelar, o que determina diferentes apresentações clínicas. Descrevemos um caso de sindrome de Fahr secundária ao hipoparatireodismo em uma mulher idosa com quadro de sonolência, dispneia e tiques.

\section{RELATO DO CASO}

Paciente T.F.O., 78 anos, sexo feminino, natural e procedente de Salvador, aposentada, com diagnóstico prévio de hipotireoidismo e depressão, foi admitida na emergência do Hospital Santa Izabel, com quadro de sonolência há 3 dias. Refere que o quadro teve início súbito e associado à dispneia em repouso, sem tosse, sem piora ao esforço ou edema de membros inferiores. Apresentou também febre não mensurada, picos isolados, sem calafrios. Não há relato de quadros previos. Negou dor precordial, lipotímia, nauseas e vômitos. $\mathrm{Na}$ história pregressa, refere episódios de crises convulsivas, que recorreram por duas vezes, com necessidade de internação para tratamento. Relata também tiques nos quatro membros, ha cerca de 2 anos, com piora progressiva. Referiu uso regular de Desvenlafaxina 100 mg/dia, Puran T4 mcg/dia, Rivotril 2 mg/dia e reposição oral de cálcio. Ao exame físico, apresentava-se sonolenta, contactante aos chamados, lúcida, orientada, porém febril $\left(\mathrm{T}: 38.1^{\circ} \mathrm{C}\right)$, taquicárdica ( $\left.\mathrm{FC}: 133 \mathrm{bpm}\right)$ e com crépitos no hemitórax direito, à ausculta respiratória. Demais sistemas sem alterações. Apresentava tiques (abalos simétricos e involuntários de grupos musculares) nos quatro membros, com predomínio em membros inferiores e sinais de Chevostek e Trousseau positivos.
Exames complementares revelaram a presença de hipocalcemia severa (Cálcio sérico: $4.6 \mathrm{mg} / \mathrm{dl}$ (VR: 8-10); Cálcio iônico: 0,64 mmol/L (VR: 1.16-1.32) e PTH: 8 pg/ ml (VR: 4-68), Vitamina D: 19,7 ng/ml (VR< 20); Magnesio: $1.4 \mathrm{mg} / \mathrm{dl}$ (VR: 1,7-2,5), compatíveis com o diagnóstico de hipoparatireoidismo primário). A tomografia de crânio revelou calcificações dos núcleos da base (Figura 1). Com o início da reposição de cálcio e vitamina D para o tratamento da hipocalcemia, a paciente evoluiu com remissão completa do quadro de tiques e desaparecimento dos sinais de Chvostek e Trousseau após 5 dias. Recebeu antibioticoterapia para infecção do trato respiratório com melhora total dos sintomas. A paciente recebeu alta com prescrição de reposição de cálcio e calcitriol e foi encaminhada para acompanhamento com endocrinologista e com diagnóstico confirmado de hipoparatireoidismo primário associado à Síndrome de Fahr.

\section{DISCUSSÃO}

Por volta de 1930, na Germânia, o neurologista Karl Theodor Fahr descreveu o primeiro caso de Síndrome de Fahr, após autópsia de um paciente de 81 anos, que apresentava crises epilepticas '.O achado de depósito de cálcio nos gânglios da base e nos vasos cerebrais explicou a presença de algumas disfunções neurológicas e psiquiátricas ${ }^{1,2}$. A sindrome de Fahr caracteriza-se pelo deposito de cálcio nas regiões perivasculares, na camada íntima e membrana basal dos vasos, seguidos de degeneração neuronal e gliose dos astrocitos com hipertrofia, hiperplasia e, finalmente, necrose ${ }^{3,4}$. Os locais mais comuns de depósito são o globo palido, talamo, núcleo denteado, cortex cerebral e cerebelo subcortical. E são associados a mecanismos distintos de sua formação, que resultam em manifestações clínicas diferenciadas ${ }^{5,6}$.

A doença acomete mais pacientes na terceira e quarta decadas de vida, sem predileçăo por sexo ${ }^{27}$ Jaworski e seus colaboradores (2017) descreveram 3 tipos de apresentação clínica da Síndrome de Fahr: início na infância (inibição do desenvolvimento mental e mortalidade precoce), início precoce, por volta dos 30 anos (sintomas predominantemente psiquiátricos), início tardio, por volta dos 50 anos (demência progressiva e desordens do movimento)

A etiologia pode ser de origem primária genética, quando envolve pessoas de uma mesma linhagem familiar e a mutação no locus 14q48, ou secundária, conhecida como Síndrome de Fahr's. Diversas outras patologias estão associadas (Figura 2), como as desordens endocrinológicas: pseudo-hipoparatiroidismo, hipoparatireodismo secundário ou primario, sendo que este último e uma das principais causas de hipocalcemia crônica e calcificação cerebral ${ }^{9}$. No caso relatado, demonstra-se um quadro de hipoparatiroidismo primário com manifestação clínica de hipocalcemia crônica e Síndrome de Fah

O hipoparatireoidismo resulta em hipocalcemia e hiperfosfatemia, cujo quadro clínico manifesta-se com tetania, insuficiência cardíaca congestiva refratária, distúrbios psiquiátricos e do estado mental ${ }^{10}$. A intensidade e o tempo de redução das concentrações dos níveis de cálcio determinam a severidade e tipo de apresentação clínica. Complicaçoes crônicas incluem catarata precoce, pseudotumor cerebral ou calcificações dos gânglios da base, que, quando bilateral e siétrica, caracteriza a Sind

A depender do local do depósito de cálcio, a Síndrome de Fahr apresenta uma variedade de sintomas neurológicos, com a demência, epilepsia, espasticidade, tetania e ate coma ${ }^{10}$. Alterações do movimento encontradas são coreia, parkinsonismo e tremores. $E$ as alterações psiquiátricas compreendem depressão, esquizofrenia e psicose. Disturibios da atenção e da cognição também estáo associados ${ }^{\circ}$. Os sinais de Chevostek e Trousseau, que incicam iritabilidad sistema excito condutor e muscular, geralmente estão presentes ao exame fisico

O diagnóstico de Síndrome de Fahr é definido pelo achado de calcificação dos núcleos da base (ou outras regióes cerebrais ou cerebelares) por exame de imagem $^{3}$. A tomografia de crânio (TC) é o método de escolha devido à maior sensibilidade do que a ressonância magnetica (RM) para a visualização de núcleos de calcificação $0^{4,8}$. Na RM identifica-se tais achados com imagem de baixa intensidade em $T 2$ e variavel em $\mathrm{T} 1^{15,6,8}$. Os locais mais acometidos são núcleo reticular, globo palido e cortex cerebral. Estudos recentes têm revelado que a RM por difusão mostrou maior sensibilidade que a TC e pode ser uma ferramenta promissora para o diagnóstico desta síndrome ${ }^{1}$. Em 2005, Manyam definiu 5 criterios para auxiliar no diagnostico da Síndrome de Fahr. 1. Calcilicação bilateral dos gânglios da base. 2. Disfunção neurológica como desordens do movimento e manifestações neuropsiquiátricas. 3 . Ausencia de anormalidades biomecânicas sugestivas de doença mitocondrial, desordem metabólica ou sistêmica. 4. Ausencia de causas tóxicas, traumáticas e infecciosas. 5. História familiar consistente e/ou comprovação genética ${ }^{3}$.

Não existe tratamento que limite a progressão da calcificação dos núcleos da base na Síndrome de Fahr ${ }^{9}$. O tratamento é baseado no alívio sintomático e melhora dos sinais clínicos'. Controle de ansiedade, depressão e reposição de cálcio são algumas das terapeuticas utilizadas. $O$ tratamento do hipoparatireoidismo e realizado com reposição de cálcio e calcitriol até atingir níveis séricos de 8-8,5mg/dl. Em casos especiais, análogos de PTH são recomendados ${ }^{10}$.

Descrevemos um caso de Síndrome de Fahr secundário ao hipoparatireoidismo primário com quadro típico de sintomas de hipocalcemia, que melhoraram apos reposição de cálcio e calcitriol. A calcificação das regiões cerebrais permanecerá e o tratamento do hipoparalireodismo proporcionara melhora sintomática e qualidade de vida.

Figuras

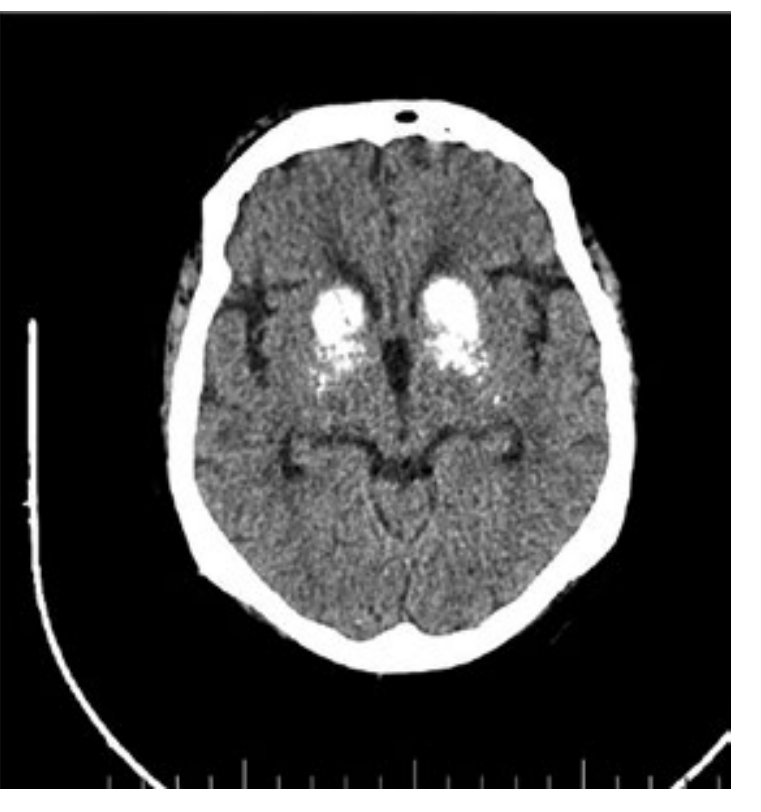

Figura 1 - Tomografia de crânio da paciente TFO, mostrando calcificação bilateral e simétrica dos núcleos da base. 


\begin{tabular}{|c|c|c|}
\hline \multicolumn{3}{|c|}{ Etiologias e quadro clínico da Síndrome de Fahr } \\
\hline Etiologias & & Quadro Clínico \\
\hline $\begin{array}{l}\text { Desordens } \\
\text { endocrino- } \\
\text { lógicas }\end{array}$ & $\begin{array}{l}\text { Hipoparatiroidismo primário } \\
\text { Hipoparatiroidismo Secundário } \\
\text { Pseudo-hipoparatireoidismo }\end{array}$ & $\begin{array}{l}\text { Tetania } \\
\text { Espasticidade } \\
\text { Mioclonias } \\
\text { Perda de Consciência } \\
\text { Depressão }\end{array}$ \\
\hline $\begin{array}{l}\text { Condições } \\
\text { neurodege- } \\
\text { nerativas do } \\
\text { adulto }\end{array}$ & $\begin{array}{l}\text { Doença do acúmulo cerebral de } \\
\text { ferro com neurodegeneração } \\
\text { Neuroferritinopatia } \\
\text { Osteodisplasia Lipo membranosa } \\
\text { Policística com Leucoencefalopa- } \\
\text { tia Esclerosante }\end{array}$ & $\begin{array}{l}\text { Fatigabilidade } \\
\text { Movimentos involun- } \\
\text { tários } \\
\text { Distonia } \\
\text { Rigidez } \\
\text { Coreia } \\
\text { Parkinsonismo }\end{array}$ \\
\hline $\begin{array}{l}\text { Doenças } \\
\text { infecciosas }\end{array}$ & $\begin{array}{l}\text { Infecções perinatais e intraute- } \\
\text { rinas } \\
\text { Síndrome de Cockayne tipo } 1 \\
\text { Síndrome de Cockayne tipo } 2\end{array}$ & $\begin{array}{l}\text { Demência } \\
\text { Retardo no desenvol- } \\
\text { vimento } \\
\text { Papiledema e hiper- } \\
\text { tensão intracraniana }\end{array}$ \\
\hline $\begin{array}{l}\text { Outras } \\
\text { causas }\end{array}$ & $\begin{array}{l}\text { Brucelose } \\
\text { Síndrome de Coat } \\
\text { Esclerose Tuberosa Complexa }\end{array}$ & $\begin{array}{l}\text { Paralisia } \\
\text { Distúrbios epilépticos } \\
\text { Psicose } \\
\text { Convulsão }\end{array}$ \\
\hline
\end{tabular}

Figura 2 - Etiologias e quadro clínico da Síndrome de Fahr. Adaptado do artigo Fahr's syndrome: literaturere view of current evidence (2017).

\section{REFERÊNCIAS}

1. Savino E, Soavi C, Capatti E, Borrelli M, Vigna GB, Passaro A, et al. Bilateral strio-pallido-dentate calcinosis (Fahr's disease): report of seven cases and revision of literature. BMC Neurol. 2016 Sep 8;16:165.

2. Saleem S, Aslam HM, Anwar M, Anwar S, Saleem M, Saleem A, et al. Fahr's syndrome: literature review of current evidence. Orphanet J Rare Dis. 2013 Oct 8;8:156.

3. Jaworski K, Styczyńska M, Mandecka M, Walecki J, Kosior DA. Fahr syndrome : an important piece of a puzzle in the differential diagnosis of many diseases. Pol J Radiol [Internet]. 2017 [cited 2018 Feb 19];82. Available from: https://ruj.uj.edu.pl/xmlui/handle/item/44505.

4. Yamada $\mathrm{M}$, Asano $\mathrm{T}$, Okamoto $\mathrm{K}$, Hayashi $\mathrm{Y}$, Kanematsu M, Hoshi $\mathrm{H}$, et al. High frequency of calcification in basal ganglia on brain computed tomography images in Japanese older adults. Geriatr Gerontol Int. 2013 Jul;13(3):706-10.

5. Kozik M, Kulczycki J. Laser-spectrographic analysis of the cation content in Fahr's syndrome. Arch Psychiatr Nervenkr. 1978 Jun 5;225(2):135-42.

6. Forensic Neuropathology and Associated Neurology I Manfred Oehmichen I Springer [Internet]. [cited 2018 Feb 19]. Available from: http://www.springer.com/ $\mathrm{gp} / \mathrm{book} / 9783642006982$
7. Morgante L, Vita G, Meduri M, Di Rosa AE, Galatioto S, Coraci MA, et al. Fahr's syndrome: local inflammatory factors in the pathogenesis of calcification. J Neurol. 1986 Feb;233(1):19-22.

8. Faria AV, Pereira IC, Nanni L. Computerized tomography findings in Fahr's syndrome. Arq Neuropsiquiatr. 2004 Sep;62(3B):789-92.

9. Sava A, Dumitrescu G, Haba D, Hodorog D, Mihailov C, Şapte E. The Fahr syndrome and the chronic lymphocytic thyroiditis. Romanian J Morphol Embryol Rev Roum Morphol Embryol. 2013;54(1):195200.

10. Shoback D. Clinical practice. Hypoparathyroidism. N Engl J Med. 2008 Jul 24;359(4):391-403.

1. Serviço de Clínica Médica do HSI

E-mail para correspondência:

adriane.spaz@hotmail.com 